\title{
The International Trauma Questionnaire: development of a self-report measure of ICD-11 PTSD and complex PTSD
}

Cloitre M, Shevlin M, Brewin CR, Bisson JI, Roberts NP, Maercker A, Karatzias T, Hyland P. The International Trauma Questionnaire: development of a self-report measure of ICD-11 PTSD and complex PTSD.

Objective: The purpose of this study was to finalize the development of the International Trauma Questionnaire (ITQ), a self-report diagnostic measure of post-traumatic stress disorder (PTSD) and complex PTSD (CPTSD), as defined in the 11 th version of the International

Classification of Diseases (ICD-11).

Method: The optimal symptom indicators of PTSD and CPTSD were identified by applying item response theory (IRT) analysis to data from a trauma-exposed community sample $(n=1051)$ and a trauma-exposed clinical sample $(n=247)$ from the United Kingdom. The validity of the optimized 12-item ITQ was assessed with confirmatory factor analyses. Diagnostic rates were estimated and compared to previous validation studies.

Results: The latent structure of the 12-item, optimized ITQ was consistent with prior findings, and diagnostic rates of PTSD and CPTSD were in line with previous estimates.

Conclusion: The ITQ is a brief, simply worded measure of the core features of PTSD and CPTSD. It is consistent with the organizing principles of the ICD-11 to maximize clinical utility and international applicability through a focus on a limited but central set of symptoms. The measure is freely available and can be found in the body of this paper.

\author{
M. Cloitre ${ }^{1,2}$ \\ C. R. Brewin 4 , M. Shevlin ${ }^{3}$ iD \\ N. P. Roberts ${ }^{6,7}$ (D) A. Maercker ${ }^{8}$ \\ T. Karatzias ${ }^{9,10}$ (iD, \\ P. Hyland ${ }^{11,12}$ iD
}

\section{Significant outcomes}

- A 12-item version of the International Trauma Questionnaire (ITQ) for the ICD-11 PTSD and CPTSD disorders was finalized and validated.

- Consistent with ICD-11 guidelines, the ITQ is (i) a brief and simply worded measure that facilitates straightforward translation and maximizes international applicability and (ii) provides a set of simple diagnostic rules to maximize ease of use in clinical and research settings.

- The ITQ is freely available in the public domain for all interested parties without any change. Further evaluation and development of the measure are needed, as is research regarding the nature, predictors, course, treatment and outcomes of PTSD and CPTSD.

\section{Limitations}

- While the community sample was drawn from a nationally representative panel, it cannot be concluded that the trauma-exposed participant sample was nationally representative.

- The participants in the clinical sample were recruited from trauma speciality clinics and may not be representative of the general help-seeking trauma-exposed population.

- Generalizability of the current findings to other countries, especially non-English speaking countries, is unknown. 
${ }^{1}$ National Center for PTSD Dissemination and Training Division, VA Palo Alto Health Care System, Palo Alto, CA, USA, ${ }^{2}$ Department of Psychiatry and Behavioural Sciences, Stanford University, Stanford, CA, USA, ${ }^{3}$ School of Psychology, Ulster University, Derry, Northern Ireland, ${ }^{4}$ Clinical Educational \& Health Psychology, University College London, London, UK, ${ }^{5}$ School of Medicine, Cardiff University, Cardiff, UK, ${ }^{6}$ Psychology and Psychological Therapies Directorate, Cardiff \& Vale University Health Board, Cardiff, UK, ${ }^{7}$ Division of Psychological Medicine \& Clinical Neurosciences, Cardiff University, Cardiff, UK, ${ }^{8}$ Department of Psychopathology and Clinical Intervention, University of Zurich, Zurich, Switzerland, ${ }^{9}$ Rivers Centre for Traumatic Stress, NHS Lothian, Edinburgh, UK, ${ }^{10}$ School of Health \& Social Care, Edinburgh Napier University, Edinburgh, UK, ${ }^{11}$ School of Business, National College of Ireland, Dublin, Ireland and ${ }^{12}$ Centre for Global Health, Trinity College Dublin, Dublin, Ireland

Key words: post-traumatic stress disorder; complex PTSD; ICD-11; the International Trauma Questionnaire; self-report

Marylène Cloitre, National Center for PTSD Dissemination and Training Division, Department of Psychiatry and Behavioural Sciences, VA Palo Alto Health Care System, Stanford University, Stanford, CA 94025, USA.

Email: marylene.cloitre@nyumc.org

Accepted for publication August 13, 2018

\section{Introduction}

The World Health Organization (WHO) published the 11th revision of the International Classification of Diseases (ICD-11) in 2018, the first major revision to the ICD in 26 years (1). The organizing principles underpinning revisions to mental disorders in ICD-11 were that disorders should have clinical utility, be focused on a limited set of core symptoms and have international applicability (2). A revised definition of post-traumatic stress disorder (PTSD), comprised of six symptoms distributed across three symptoms clusters (Reexperiencing in the here and now, avoidance of traumatic reminders and a sense of threat), is included within the category of 'Disorders Specifically Associated with Stress'. A sibling diagnosis of complex PTSD (CPTSD) is also included in this category and is comprised of the core PTSD symptom clusters plus three additional symptom clusters (affective dysregulation, negative self-concept and disturbances in relationships) that collectively represent 'Disturbances in SelfOrganization' (DSO) (3). Brewin et al. (4) reviewed the existing literature on ICD-11 PTSD and CPTSD and found strong support for their construct validity.

Unlike the DSM (5), the ICD does not necessarily provide a defined list of specific symptoms necessary for a diagnosis of a given disorder. Rather, the ICD provides a narrative description of the 'definition of a disorder along with a list of that disorder's essential (required) features (6). This broad formulation sets a framework for the general understanding of a disorder. However, the absence of specific symptoms and diagnostic criteria creates ambiguity and potential problems in establishing a shared understanding of the meaning and presentation of a disorder among both clinicians and researchers. In an attempt to operationalize the narrative descriptions of ICD-11 PTSD and CPTSD provided by the WHO (6), researchers including members of the "Working Group for Disorders Specifically Associated with
Stress' developed a preliminary-stage, self-report measure called the International Trauma Questionnaire (ITQ) (7), along with a defined set of diagnostic criteria. The development of the PTSD items was influenced by the work of Brewin et al. (8), and the development of the DSO items was based on the results of the DSM-IV field trials which assessed the most frequently reported CPTSD symptoms (9), and the results of a consensus survey among expert clinicians who were asked to identify the most frequent and most impairing CPTSD symptoms (10). The preliminary-stage version of the ITQ included 28 test items, and multiple studies have shown that its latent structure reflects the distinction between PTSD and DSO symptomatology and provided support for the factorial, discriminant, concurrent, predictive and cross-cultural validity of PTSD and CPTSD $(4,11$, 12) (see Appendix 1 for a list of all ITQ items). However, to align with the organizing principle of ICD-11 that disorders should focus on a limited but central set symptoms, the goal of the current study is to abbreviate the ITQ to a final set of 12 items so that each PTSD and DSO cluster is represented by two items.

Aims of the study

To achieve this goal, the psychometric properties of all (dichotomously scored) ITQ items were assessed using item response theory (IRT) models. Although much of the existing ITQ psychometric research has employed factor analysis models (11, 12), IRT models are more appropriate to assess the performance of indicators when their purpose is to identify the presence of a symptom. In relation to the final selection of PTSD items, the psychometric performance of the two commonly used re-experiencing items (RE1: nightmares and RE2: flashbacks) would have to be found to be poor to consider replacing either (or both) with alternative test items. The criteria for the selection of the DSO items were that (i) the Affective Dysregulation cluster should include one 'hyperactivation' item 


\section{Cloitre et al.}

and one 'deactivation' item (see 13); (ii) items with higher discrimination would be preferred; and (iii) items that have excessively high or low thresholds for endorsement would be rejected. Following the selection of the final set of 12 items for the optimized version of the ITQ, diagnostic rates for ICD-11 PTSD and CPTSD were estimated and compared to those from the previously used diagnostic algorithm based on the preliminary-stage version of the ITQ. The latent structure of the optimized ITQ was assessed using confirmatory factor analysis (CFA), and diagnostic groups were compared in terms of their levels of lifetime interpersonal trauma.

\section{Material and methods}

Participants and procedures

The current study was based on two distinct samples drawn from the adult population of the United Kingdom (UK). Sample 1 was a community sample drawn from an existing online research panel that is representative of the entire UK adult population. Panel members were randomly recruited through probability-based sampling, and inclusion criteria for sample selection in this case were that respondents (a) had been born in the UK, (b) were aged 18 years or older at the time of the survey and (c) screened positive for at least one lifetime traumatic event (assessed using the Life Events Checklist, described below). Ethical approval was granted by the ethical review board of the institution to which the last author is affiliated. No inducements or incentives were offered for participation. In total, 2653 panel members were assessed to meet the inclusion criteria and 1051 people qualified as valid cases (selection rate $=39.6 \%$ ). There were no missing data. This mean age of the sample was 47.18 years $(\mathrm{SD}=15.00, \quad$ range $=18-90$ years $)$, and $68.4 \%$ $(n=719)$ of participants were female. The majority of individuals indicated that they were in a committed relationship $(70.4 \%, n=740)$, did not have children under the age of 16 years $(67.5 \%$, $n=709$ ), had completed third-level education $(62.7 \%, n=659)$ and were in full- or part-time employment $(58.5 \%, n=615)$. A number of participants indicated that they had emigrated at some point in their lifetime $(17.8 \%, n=187)$.

Sample 2 was a clinical sample, and participants were recruited in an opportunistic manner from two treatment centres in the UK that provide psychological treatment for trauma-exposed persons $(N=247)$. No incentives or inducements were used to recruit participants, and participation did not determine access to care. Ethical approval for this data collection was provided by the relevant local research ethics committees. The mean age of the sample was 42.07 years $(\mathrm{SD}=12.96$, range $=18$ 71 years $)$, and $68.0 \%(n=168)$ were female. The majority of the sample indicated that they were unemployed $(52.8 \%, n=130)$, not in a committed relationship $(68.5 \%, n=167)$, and had completed third-level education $(52.6 \%, n=130)$. Full data were available for this sample.

\section{Measures}

Traumatic exposure. The Life Events Checklist for DSM-5 (LEC-5) (14) was used in both samples to assess lifetime traumatic exposure. Participants were asked to indicate on a 'Yes' (1) or 'No' (0) basis if they had directly experienced 16 traumatic events plus any other traumatic event not listed. A total score was calculated for each sample ranging from 0 to 17. The mean number of lifetime traumas in the community sample was 3.36 $(\mathrm{Mdn}=3.00, \mathrm{SD}=2.70$, range $=1-17)$, and the most commonly experienced trauma was the sudden and unexpected death of someone close to you $(56.6 \%, n=595)$. This event was also the most commonly reported 'most distressing traumatic event' $(29.4 \%, n=309)$. Among the clinical sample, the mean number of lifetime traumas was 6.68 $(\mathrm{Mdn}=6.00, \mathrm{SD}=3.12$, range $=1-17)$, the most commonly experienced trauma was physical assault $(86.6 \%, n=214)$, and sexual assault was the most commonly reported 'most distressing traumatic event' $(23.5 \%, n=58)$. Following Ehring and Quack's (15) recommendations, a total score of interpersonal trauma (physical assault, assault with a weapon, sexual assault, other unwanted or uncomfortable sexual experiences, combat or exposure to a war-zone, captivity, serious injury and/or harm and/or death you caused to someone else) was calculated where scores ranged from 0 to 7 .

ICD-11 PTSD and CPTSD. The preliminary-stage version of the ITQ (7) used in this study included 12 PTSD items and 16 DSO items. There were eight re-experiencing items including two that have been consistently used (RE1 and RE2) and six test indicators (RE3-RE8), some of which were taken from the Dissociative Symptoms Scale (16). There were two items measuring avoidance (AV1, AV2) and sense of threat (TH1, TH2) symptoms. The avoidance and sense of threat items were adapted from the Posttraumatic Stress Disorder Checklist for DSM-5 (PCL-5) (17). There were nine Affective Dysregulation items (five 'hyperactivation' [AD1- 
AD5] and four 'deactivation' [AD6-AD9] items), four Negative Self-Concept (NSC1-NSC4) items and three Disturbances in Relationship (DR1DR3) items. Additionally, three items measure functional impairment (social, occupational and other important areas of life) associated with the PTSD and DSO symptoms respectively. Internal reliability was assessed by Cronbach's alpha $(\alpha)$, and within the community sample, $\alpha$ for all PTSD and DSO subscales were $\geq 0.77$, with the exception of the avoidance items which were slightly lower than desirable $(\alpha=0.67)$. In the community sample, reliabilities for all PTSD and DSO subscales were satisfactory; all $\alpha \mathrm{s} \geq 0.79$.

The ITQ items were measured using a five-point Likert scale ranging from 'Not at all' (0) to 'Extremely' (4). Following standard practice in trauma research $(18,19)$, scores $\geq 2$ ('Moderately') were used to indicate the presence of a symptom. All analyses were based on these dichotomized items. Two diagnostic algorithms for ICD-11 PTSD and CPTSD were used in this study. The first is consistent with the diagnostic algorithm that has been used in all prior studies utilizing the preliminarystage version of the ITQ (see 11, 12). The second is based on the optimized version of the ITQ. Under this algorithm, diagnosis of PTSD requires the endorsement of one of two symptoms from each PTSD cluster, plus endorsement of functional impairment associated with these symptoms. Diagnosis of CPTSD requires the endorsement of one of two symptoms from each of the six PTSD and DSO clusters, plus endorsement of functional impairment associated with these symptoms. The ICD-11 taxonomic structure dictates that a person may only receive a diagnosis of PTSD or CPTSD, but not both.

\section{Data analysis}

Analysis for this study consisted of two linked phases. In Phase 1, endorsement rates were calculated for all ITQ items, and 1- and 2-parameter binary logistic IRT models were estimated for the PTSD and DSO items separately. Mplus 7.4 (20) was used to specify and estimate the model parameters using robust maximum-likelihood. For the 2-parameter model, discrimination and difficulty parameters were estimated for all items. The discrimination parameter is the logistic regression that relates the latent variable, theta $\theta$ (with a mean of 0 and a variance of 1 ), to the binary indicator where higher values indicate increased discriminatory power. The difficulty parameter represents 'cut-points' on the underlying trait $(\theta)$. Mplus estimates these parameters as thresholds, and these were converted into difficulty estimates that represent the level of $\theta$ where an individual has a probability of 0.50 of endorsing the indicator. A 1-parameter model was also tested where the item discrimination parameters were constrained to be equal for indicators loading on each latent variable. This is 'within cluster equality' where the discrimination parameters for the indicators for each symptom cluster were constrained equal, but no constraints were imposed across clusters. The Akaike information criterion (AIC), the Bayesian information criterion (BIC) and the sample size-adjusted Bayesian information criterion (ssaBIC) were used to evaluate the models. The model with the lowest BIC value was considered to be the better model, and a difference of $\geq 10$ was considered to be indicative of a 'significant' difference (21). On the basis of parsimony, the 1-parameter model was selected unless the information criteria indicated that the 2-parameter model was superior. With such a large number of indicators, some violations of the assumptions of IRT were likely, particularly local independence, but this model provides easily interpretable parameters that could help inform the process of item selection. Therefore, the IRT modelling at this stage was not used as a method to identify the best performing items; rather, it was used to identify any potentially problematic items with obviously poor performance such as excessively high or low difficulty and/or poor discrimination. The information on endorsement rates, discrimination/difficulty and clinical relevance was used collectively to identify PTSD and DSO indicators that could be used for the 12-item, optimized version of the ITQ.

In Phase 2, the psychometric and diagnostic performance of the optimized ITQ was assessed. This involved (i) assessing the latent structure of the ITQ using CFA based IRT model; (ii) testing for differential item functioning based on a multigroup IRT model; (iii) calculating the diagnostic rates of ICD-11 PTSD and CPTSD and comparing these findings to the diagnostic rates produced using the preliminary-stage version of the ITQ; and (iv) testing whether there were significant differences in lifetime interpersonal trauma exposure across the diagnostic categories. To assess the latent structure of the optimized ITQ, two models identified in prior validation studies were evaluated $(11,12)$. Model 1 is a correlated six-factor model (Reexperiencing, avoidance, sense of threat, affective dysregulation, negative self-concept, and disturbances in relationships) where each factor is measured by two items. Model 2 is a two-factor second-order model whereby the first-order factor correlations are explained by two correlated 


\section{Cloitre et al.}

second-order factors: PTSD and DSO. These models were estimated using the robust weighted least squares estimator (WLSMV) with a nonlinear probit link based on the tetrachoric correlation matrix of latent continuous response variables and delta parameterization. The scale of the latent variables was set by fixing the first loading of each latent variable at 1 thereby allowing the factor variances to vary across the groups. Model fit was evaluated in relation to a number of goodness-of-fit indices, and standard criteria were used to determine the model fit (22): a nonsignificant chi-square $\left(\chi^{2}\right)$ result indicates good model fit; Comparative Fit Index (CFI) and the Tucker-Lewis Index (TLI) values $\geq 0.90$ and $\geq 0.95$ reflect acceptable and excellent model fit respectively; and root mean square error of approximation (RMSEA) values $\leq 0.08$ and $\leq 0.05$ indicate acceptable and excellent model fit respectively.

Subsequent models that tested for 'configural' and 'scalar' invariance were fitted to the correlated six-factor model. The configural model specified a multigroup model where the loadings were free to vary across the clinical and community groups. The scalar model placed equality constraints on the loadings across the groups. Thresholds were invariant across groups, and the latent variable means in the community group were fixed to zero, and the latent variable means for clinical group were estimated. The relative fit of the models was tested using the DIFFTEST (23). It has been shown that overall WLSMV-based model fit statistics are not sensitive enough to identify potential violations of local independence (24) that can result in biased parameter estimates. To identify potential violations of local independence, the solution from the multigroup analysis was examined using the modification indices (MI) and the expected parameter change (EPC) parameters. The MIs were used to identify potential correlated residual errors that should be included in the model; a cut-off value of 10 was used as MIs have been shown to increase the risk of type 1 errors with large samples (25). The EPC estimates the expected value of a fixed parameter if it was a freely estimated parameter in the model. The EPC for the residual correlations (which is analogous to Yen's $\mathrm{Q}_{3}$ ) (26) was inspected, and values $>0.20$ would be indicative of local independence violations (27). Following the guidance of Saris, Satorra and van der Veld (28), MIs and EPCs were interpreted in combination.

Finally, the diagnostic groups (no diagnosis, PTSD and CPTSD) identified by the optimized ITQ were compared in relation to their mean levels of lifetime interpersonal trauma using a one-way between-group analysis of variance (ANOVA). The Tukey HSD test was used for post hoc pairwise comparisons, and overall effect sizes were calculated using eta-squared $\left(\eta^{2}\right)$. Based on Cohen's guidelines (29), $\eta^{2}$ values from 0.01 to 0.05 reflect a small effect, values from 0.06 to 0.13 indicate a medium effect, and values $\geq 0.14$ indicate a large effect.

\section{Results}

Phase 1 results: Binary logistic IRT model results

Table 1 reports the fit statistics for the IRT models of the PTSD and DSO items in both samples. The BIC value was lower for the 1-parameter model compared to the 2-parameter model in each case, indicating that the items were equivalent in discriminatory power, except for the DSO model based on the data from the community sample.

Tables 2 and 3 report the endorsement rates and IRT parameters for the PTSD and DSO items in both samples. The endorsement rates for the two commonly used re-experiencing items (RE1 and RE2) were slightly lower than the endorsement rates for the avoidance and sense of threat items. However, neither item possessed excessively high (RE7) or low (RE4) endorsement rates. Furthermore, RE1 and RE2 produced satisfactory discrimination and difficulty parameters, and as such, there was no evidence to indicate the need to replace either item. Therefore, RE1 and RE2 were selected for inclusion in the optimized ITQ alongside AV1, AV2, TH1 and TH2.

The Affective Dysregulation items were inspected in order to select one 'hyperactivation' item (AD1-AD5) and one 'deactivation' item (AD6-AD9). The threshold/difficulty parameters of AD4, AD5, AD8 and AD9 were deemed to be excessively high relative to the other items within these clusters and were consequently rejected. The remaining items performed similarly in both

Table 1. Fit statistics for the item response theory models of PTSD and DSO symptoms

\begin{tabular}{lccrrr}
\hline Sample & Scale & Model & AIC & \multicolumn{1}{c}{ BIC } & \multicolumn{1}{c}{ ssaBIC } \\
\hline Community & PTSD & 1-parameter & 9138.979 & 9228.214 & 9171.043 \\
& & 2-parameter & 9129.527 & 9263.379 & 9177.623 \\
& DSO & 1-parameter & 13474.879 & 13583.944 & 13514.069 \\
& & 2-parameter & 13357.405 & 13530.918 & 13419.752 \\
Clinical & PTSD & 1-parameter & 2463.162 & 2526.331 & 2469.271 \\
& & 2-parameter & 2455.515 & 2550.268 & 2464.678 \\
& DSO & 1-parameter & 3586.520 & 3667.236 & 3594.327 \\
& & 2-parameter & 3571.794 & 3698.132 & 3584.012 \\
\hline
\end{tabular}

AIC, Akaike information criterion; BIC, Bayesian information criterion; DSO, Disturbances in Self-Organization; PTSD, post-traumatic stress disorder; ssaBIC, sample size-adjusted BIC. 
The International Trauma Questionnaire

Table 2. Endorsement rates and item response parameters for all ITQ item for the community sample ( $N=1051)$

\begin{tabular}{|c|c|c|c|c|c|}
\hline \multirow[b]{2}{*}{ Indicator } & \multicolumn{2}{|c|}{ Endorsement } & \multirow[b]{2}{*}{ Discrimination (SE) } & \multirow[b]{2}{*}{ Threshold (SE) } & \multirow[b]{2}{*}{ Difficulty (SE) } \\
\hline & $N$ & $\%$ & & & \\
\hline \multicolumn{6}{|l|}{ PTSD symptoms } \\
\hline RE1. Upsetting dreams & 282 & 26.8 & $3.89(0.17)$ & $2.59(0.18)$ & $0.666(0.05)$ \\
\hline RE2. Reliving event in the here and now & 334 & 31.8 & $3.89(0.17)$ & $1.99(0.17)$ & $0.512(0.04)$ \\
\hline RE3. Being reminded then spacing out & 319 & 30.4 & $3.89(0.17)$ & $2.16(0.17)$ & $0.555(0.04)$ \\
\hline RE4. Moments when lose control and act as in past & 197 & 18.7 & $3.89(0.17)$ & $3.73(0.20)$ & $0.960(0.05)$ \\
\hline RE5. Memory so strong lose track of surroundings & 272 & 25.9 & $3.89(0.17)$ & $2.71(0.18)$ & $0.697(0.05)$ \\
\hline RE6. React to others as back in the past & 242 & 23.0 & $3.89(0.17)$ & $3.01(0.19)$ & $0.796(0.05)$ \\
\hline RE7. Upset by reminders & 482 & 45.9 & $3.89(0.17)$ & $0.45(0.16)$ & $0.117(0.04)$ \\
\hline RE8. Flashbacks even for a moment & 283 & 26.9 & $3.89(0.17)$ & $2.58(0.18)$ & $0.663(0.05)$ \\
\hline AV1. Internal reminders & 396 & 37.7 & $6.32(0.58)$ & $2.03(0.29)$ & $0.322(0.04)$ \\
\hline AV2. External reminders & 364 & 34.6 & $6.32(0.58)$ & $2.55(0.32)$ & $0.404(0.04)$ \\
\hline TH1. Being on guard & 378 & 36.0 & $6.53(0.62)$ & $2.38(0.33)$ & $0.364(0.04)$ \\
\hline TH2. Jumpy/startled & 310 & 29.5 & $6.53(0.62)$ & $3.57(0.39)$ & $0.546(0.04)$ \\
\hline \multicolumn{6}{|l|}{ DSO symptoms } \\
\hline AD1. Intense reactions & 432 & 41.1 & $2.65(0.20)$ & $0.738(0.13)$ & $0.278(0.05)$ \\
\hline AD2. Long time to calm down & 450 & 42.8 & $2.78(0.21)$ & $0.623(0.13)$ & $0.223(0.05)$ \\
\hline AD3. Feelings easily hurt & 544 & 51.8 & $2.21(0.17)$ & $-0.108(0.11)$ & $-0.049(0.05)$ \\
\hline AD4. Uncontrollable anger & 299 & 28.4 & $2.53(0.19)$ & $1.759(0.15)$ & $0.695(0.05)$ \\
\hline AD5. Reckless behaviour & 195 & 18.6 & $2.50(0.22)$ & $2.724(0.21)$ & $1.087(0.06)$ \\
\hline AD6. Numb & 379 & 36.1 & $3.79(0.33)$ & $1.535(0.19)$ & $0.405(0.04)$ \\
\hline AD7. Difficulty feeling pleasure & 358 & 34.1 & $3.78(0.32)$ & $1.753(0.20)$ & $0.463(0.04)$ \\
\hline AD8. World is distant & 333 & 31.7 & $4.54(0.45)$ & $2.367(0.28)$ & $0.521(0.04)$ \\
\hline AD9. Feeling outside of body & 265 & 25.2 & $4.58(0.47)$ & $3.295(0.34)$ & $0.718(0.04)$ \\
\hline NSC1. Failure & 381 & 36.3 & $6.64(0.91)$ & $2.488(0.41)$ & $0.374(0.04)$ \\
\hline NSC2. Worthless & 363 & 34.5 & $8.41(1.43)$ & $3.516(0.66)$ & $0.418(0.04)$ \\
\hline NSC3. Shame & 372 & 35.4 & $6.37(0.70)$ & $2.545(0.34)$ & $0.399(0.04)$ \\
\hline NSC4. Guilt & 479 & 45.6 & $3.64(0.29)$ & $0.478(0.15)$ & $0.131(0.04)$ \\
\hline DR1. Feel cut-off from others & 424 & 40.3 & $5.69(0.74)$ & $1.538(0.28)$ & $0.270(0.04)$ \\
\hline DR2. Difficulty staying close to others & 416 & 39.6 & $4.54(0.48)$ & $1.344(0.22)$ & $0.296(0.04)$ \\
\hline DR3. Avoid relationships & 333 & 31.7 & $2.75(0.23)$ & $1.571(0.15)$ & $0.569(0.05)$ \\
\hline
\end{tabular}

$A D$, affective dysregulation; Av, avoidance; DR, disturbances in relationships; DSO, Disturbances in Self-Organization; NSC, negative self-concept; PTSD, post-traumatic stress disorder; Re, re-experiencing in the here and now; SE, standard error; TH, sense of threat.

samples; however, AD2 (hyperactivation) and AD6 (deactivation) showed the highest discrimination parameters in the community sample and satisfactory endorsement rates in the clinical sample and were judged to possess good clinical relevance. Therefore, AD2 and AD6 were selected to represent the Affective Dysregulation cluster.

With respect to the Negative Self-Concept items, the endorsement rate for NSC4 was considered excessively high relative to the other items in this cluster and was therefore rejected. NSC1-NSC3 performed similarly across both samples, and as such, any two of these three items could have been selected. NSC1 and NSC2 have been consistently used to represent this symptom cluster in prior studies (19), both items are very simply worded, and both items were judged to possess good clinical relevance. Therefore, NSC1 and NSC2 were selected to represent the Negative Self-Concept cluster.

With respect to the Disturbances in Relationships cluster, DR3 was deemed to possess excessively low discrimination and excessively high difficulty parameters relative to the other items in this cluster and was consequently rejected. Therefore, DR1 and DR2 were selected to represent the Disturbances in Relationships cluster. The 12-item, optimized version of the ITQ is presented in Appendix 2.

Phase 2: Diagnostic and psychometric performance of the optimized ITO

The CFA results of the optimized ITQ are presented in Table 4. The first- and second-order models fitted the data from the community and clinical samples extremely well. The CFI, TLI and RMSEA values all suggested excellent model fit for the first- and second-order models within both samples. The only exception was the chi-square test; however, this should not lead to model rejection as the power of the chisquare is positively related to sample size and tends to reject models based on large sample sizes (30).

The model with configural invariance had acceptable model fit. There were no MIs $>10$, and 


\section{Cloitre et al.}

Table 3. Endorsement rates and item response parameters for all item indicators for the clinical sample $(N=247)$

\begin{tabular}{|c|c|c|c|c|c|}
\hline \multirow[b]{2}{*}{ Indicators } & \multicolumn{2}{|c|}{ Endorsement } & \multirow[b]{2}{*}{ Discrimination (SE) } & \multirow[b]{2}{*}{ Threshold (SE) } & \multirow[b]{2}{*}{ Difficulty (SE } \\
\hline & N & $\%$ & & & \\
\hline \multicolumn{6}{|l|}{ PTSD symptoms } \\
\hline RE1. Upsetting dreams & 185 & 74.9 & $2.42(0.21)$ & $-1.913(0.26)$ & $-0.789(0.12)$ \\
\hline RE2. Reliving event in the here and now & 187 & 75.7 & $2.42(0.21)$ & $-1.980(0.25)$ & $-0.817(0.12)$ \\
\hline RE3. Being reminded then spacing out & 196 & 79.4 & $2.42(0.21)$ & $-2.356(0.27)$ & $-0.972(0.13)$ \\
\hline RE4. Moments when lose control and act as in past & 107 & 43.7 & $2.42(0.21)$ & $0.470(0.23)$ & $0.194(0.10)$ \\
\hline RE5. Memory so strong lose track of surroundings & 156 & 63.2 & $2.42(0.21)$ & $-0.928(0.23)$ & $-0.383(0.10)$ \\
\hline RE6. React to others as back in the past & 138 & 56.6 & $2.42(0.21)$ & $-0.457(0.23)$ & $-0.188(0.10)$ \\
\hline RE7. Upset by reminders & 220 & 89.1 & $2.42(0.21)$ & $-3.678(0.34)$ & $-1.517(0.16)$ \\
\hline RE8. Flashbacks even for a moment & 186 & 75.6 & $2.42(0.21)$ & $-1.960(0.25)$ & $-0.808(0.12)$ \\
\hline AV1. Internal reminders & 211 & 85.4 & $1.64(0.40)$ & $-2.499(0.36)$ & $-1.525(0.25)$ \\
\hline AV2. External reminders & 211 & 85.4 & $1.64(0.40)$ & $-2.506(0.36)$ & $-1.530(0.25)$ \\
\hline TH1. Being on guard & 213 & 86.2 & $3.03(0.60)$ & $-3.815(0.64)$ & $-1.260(0.13)$ \\
\hline TH2. Jumpy/startled & 209 & 84.6 & $3.03(0.60)$ & $-3.560(0.60)$ & $-1.176(0.13)$ \\
\hline \multicolumn{6}{|l|}{ DSO symptoms } \\
\hline AD1. Intense reactions & 206 & 83.4 & $1.428(0.13)$ & $-2.155(0.21)$ & $-1.509(0.19)$ \\
\hline AD2. Long time to calm down & 222 & 89.9 & $1.428(0.13)$ & $-2.879(0.25)$ & $-2.017(0.24)$ \\
\hline AD3. Feelings easily hurt & 208 & 84.2 & $1.428(0.13)$ & $-2.232(0.23)$ & $-1.563(0.18)$ \\
\hline AD4. Uncontrollable anger & 139 & 56.3 & $1.428(0.13)$ & $-0.331(0.17)$ & $-0.232(0.12)$ \\
\hline AD5. Reckless behaviour & 106 & 42.9 & $1.428(0.13)$ & $0.405(0.17)$ & $0.283(0.12)$ \\
\hline AD6. Numb & 189 & 76.5 & $1.428(0.13)$ & $-1.587(0.20)$ & $-1.112(0.15)$ \\
\hline AD7. Difficulty feeling pleasure & 181 & 73.3 & $1.428(0.13)$ & $-1.357(0.19)$ & $-0.951(0.15)$ \\
\hline AD8. World is distant & 204 & 82.6 & $1.428(0.13)$ & $-2.080(0.21)$ & $-1.457(0.18)$ \\
\hline AD9. Feeling outside of body & 170 & 68.8 & $1.428(0.13)$ & $-1.066(0.18)$ & $-0.747(0.14)$ \\
\hline NSC1. Failure & 190 & 76.9 & $4.532(0.58)$ & $-1.181(0.15)$ & $-0.795(0.09)$ \\
\hline NSC2. Worthless & 182 & 73.7 & $4.532(0.58)$ & $-3.602(0.55)$ & $-0.684(0.09)$ \\
\hline NSC3. Shame & 194 & 78.5 & $4.532(0.58)$ & $-3.099(0.53)$ & $-0.853(0.10)$ \\
\hline NSC4. Guilt & 214 & 86.6 & $4.532(0.58)$ & $-3.864(0.60)$ & $-1.186(0.11)$ \\
\hline DR1. Feel cut-off from others & 214 & 86.6 & $2.915(0.41)$ & $-5.374(0.76)$ & $-1.293(0.13)$ \\
\hline DR2. Difficulty staying close to others & 194 & 78.5 & $2.915(0.41)$ & $-3.769(0.49)$ & $-0.911(0.11)$ \\
\hline DR3. Avoid relationships & 178 & 72.1 & $2.915(0.41)$ & $-2.655(0.37)$ & $-0.668(0.10)$ \\
\hline
\end{tabular}

$A D$, affective dysregulation; Av, avoidance; DR, disturbances in relationships; DSO, Disturbances in Self-Organization; NSC, negative self-concept; PTSD, post-traumatic stress disorder; Re, re-experiencing in the here and now; SE, standard error; $\mathrm{TH}$, sense of threat.

Table 4. Fit statistics, diagnostic rates and multigroup ITR results for the optimized ITQ in the community and clinical samples

\begin{tabular}{|c|c|c|c|c|c|c|c|c|c|}
\hline Sample & $\chi^{2}$ & df & $P$ & $\mathrm{CFI}$ & TLI & RMSEA $(90 \%$ CI) & PTSD diagnosis & CPTSD diagnosis & Total \\
\hline \multicolumn{10}{|l|}{ Community } \\
\hline First-order model & 64.587 & 39 & 0.006 & 0.999 & 0.998 & $0.025(0.013-0.036)$ & \multirow[t]{2}{*}{$5.3 \%(n=56)$} & \multirow[t]{2}{*}{$12.9 \%(n=136)$} & \multirow[t]{2}{*}{$18.3 \%(n=192$} \\
\hline Second-order model & 104.036 & 47 & $<0.001$ & 0.998 & 0.997 & $0.034(0.025-0.043)$ & & & \\
\hline \multicolumn{10}{|l|}{ Clinical } \\
\hline First-order model & 62.822 & 39 & 0.009 & 0.987 & 0.979 & $0.050(0.025-0.072)$ & \multirow[t]{2}{*}{$14.6 \%(n=36)$} & \multirow[t]{2}{*}{$61.1 \%(n=151)$} & \multirow[t]{2}{*}{$75.7 \%(n=187$} \\
\hline Second-order model & 68.123 & 47 & 0.024 & 0.989 & 0.984 & $0.043(0.016-0.064)$ & & & \\
\hline Configural invariance & 128.505 & 84 & 0.001 & 0.998 & 0.998 & $0.029(0.018-0.038)$ & & & \\
\hline Scalar invariance & 142.132 & 90 & 0.000 & 0.998 & 0.997 & $0.030(0.020-0.039)$ & & & \\
\hline
\end{tabular}

$\chi^{2}$, chi-square; $\mathrm{CFI}$, comparative fit indices; $\mathrm{df}$, degrees of freedom; $P$, statistical significance; RMSEA ( $\left.90 \% \mathrm{CI}\right)$, root mean square of approximation (90\% confidence intervals); TLI, Tucker-Lewis Index.

the largest residual correlation EPC was -0.09 (for AD1 and DR2). The model with scalar invariance also fitted the data, but was a significantly poorer fit than the configural invariance model according to the DIFFTEST $\left(\Delta \chi^{2}=13.97, \Delta \mathrm{df}=6\right.$, $P=0.030)$ although the differences in the CFI/TLI and the RMSEA were very small. The only model parameter with a MI $>10$ was for the residual correlation between DR1 and AD2 $(M I=10.20)$; however, the associated EPC was -0.11 indicating that including this correlated residual would be unlikely to significantly bias the model parameters. The factor means for the clinical group were all statistically significant indicating, as expected, significantly higher levels of PTSD and DSO for this group. Based on these analyses, it can be concluded that the optimized ITQ performs equally well for the clinical and community groups as there is no evidence of differential item functioning. 
The ICD-11 PTSD and CPTSD diagnostic rates are also presented in Table 4 . In total, $18.3 \%$ $(n=192)$ of the community sample met the criteria for a diagnosis of either PTSD or CPTSD. More specifically, $5.3 \%(n=56)$ met the criteria for a PTSD diagnosis and $12.9 \%(n=136)$ met the criteria for a CPTSD diagnosis. Relative to the diagnostic algorithm for the preliminary version of the ITQ, the optimized ITQ slightly increased the number of CPTSD cases $(12.9 \%$ vs. $10.6 \%)$.

Among the clinical sample, $75.7 \%(n=187)$ met the criteria for a diagnosis of either PTSD or CPTSD, with $14.6 \%(n=36)$ meeting the criteria for a PTSD diagnosis and $61.1 \%(n=151)$ meeting the criteria for a CPTSD diagnosis. Consistent with the community sample results, the optimized ITQ produced slightly more CPTSD cases compared to the diagnostic algorithm for the preliminary version $(61.1 \%$ vs. $56.3 \%)$.

The results of the one-way between-group ANOVA tests are reported in Table 5. There were significant differences in the mean number of lifetime interpersonal traumas across the diagnostic groups [(i) no diagnosis, (ii) PTSD diagnosis and (iii) CPTSD diagnosis] in the community $[F(2$, 1048) $\left.=12.89, P<0.001, \eta^{2}=0.02\right]$ and clinical $\left[F(2,244)=10.73, P<.001, \eta^{2}=0.08\right]$ samples. Post hoc comparisons using the Tukey HSD test indicated that for both samples, those with a CPTSD diagnosis experienced significantly more interpersonal traumas than those with no diagnosis. Additionally, for the clinical sample, those with a CPTSD diagnosis experienced significantly more interpersonal traumas than those with a PTSD diagnosis.

\section{Discussion}

The primary aim of this study was to finalize the development of the ITQ so that the ICD-11

Table 5. One-way between-group ANOVA results for lifetime interpersonal trauma exposure in the community and clinical samples

\begin{tabular}{llrrrrr}
\hline & Group & $n$ & $\mathrm{M}$ & SD & $F$ & $\eta^{2}$ \\
\hline $\begin{array}{l}\text { Community sample } \\
\text { Lifetime interpersonal } \\
\text { trauma }\end{array}$ & No diagnosis & 859 & 1.19 & 1.36 & $12.89^{*}$ & 0.02 \\
& & & & & & \\
& PTSD & 56 & 1.57 & 1.58 & & \\
$\begin{array}{l}\text { Clinical sample } \\
\text { Lifetime interpersonal } \\
\text { trauma }\end{array}$ & No diagnosis & 60 & 2.70 & 1.58 & $10.73^{*}$ & 0.08 \\
& & & & & & \\
& PTSD & 36 & 2.61 & 1.55 & & \\
& CPTSD & 151 & 3.55 & 1.39 & & \\
\hline
\end{tabular}

$\eta^{2}$, eta-squared $(0.01-0.05=$ small effect, $0.06-0.13=$ medium effect, $\geq 0.14=$ large effect); $\mathrm{M}$, mean; SD, standard deviation; $* p<0.001$; models have two degrees of freedom; lifetime interpersonal trauma ranges from 0 to 7. narrative descriptions of PTSD and CPTSD could be effectively operationalized for research and clinical purposes. This involved selecting a final set of 12 symptom indicators for that best represented the symptom clusters of PTSD (re-experiencing, avoidance and sense of threat) and DSO (affective dysregulation, negative self-concept and disturbances in relationships). In line with the WHO's organizing principles for the $\operatorname{ICD}-11(2,6)$, the optimized ITQ represents a self-report diagnostic measure of PTSD and CPTSD which captures a limited but core set of symptoms using simply worded items which facilitate translation and thus maximize international applicability. Furthermore, the ITQ includes a simple and quick diagnostic algorithm which maximizes clinical (and research) utility. Importantly, consistent with the WHO principles of open science, the ITQ is made freely available in the public domain to all interested parties. It is our hope that researchers and clinicians from around the world will now begin to routinely use this measure of ICD-11 PTSD and CPTSD so as to continue to develop the evidence base not only for the scale's psychometric properties, but more importantly, to advance knowledge regarding the nature, predictors, course, treatment and outcomes of PTSD and CPTSD.

In the community sample, approximately one-in-five people $(18.3 \%)$ met the criteria for a diagnosis of PTSD or CPTSD, while three-in-four people $(75.7 \%)$ in the clinical sample met the criteria for a diagnosis of PTSD or CPTSD. In both samples, the prevalence of CPTSD was higher than PTSD, and although this is expected in populations who have been multiply traumatized (11), it is important that future research tests the hypothesis that '... community rates of PTSD are higher than CPTSD while the reverse relationship obtains in trauma specialty clinics' (8). A complicating factor in testing this hypothesis is that evidence from nationally representative surveys has shown that exposure to multiple traumas can be as common, and often more common, than single exposure. Scott et al. (31) showed that using a standardized assessment of lifetime traumatic event exposure across 14 countries, multiple rather than single exposure was more common. Given that current and past findings (18) have shown that multiple trauma exposure can be more strongly associated with CPTSD than PTSD, it may be that the prevalence of CPTSD is also higher in the general population. This remains to be determined; however, the availability of the optimized ITQ now permits this work to be undertaken.

The psychometric and diagnostic results for the optimized ITQ were encouraging. The CFA 


\section{Cloitre et al.}

results were consistent with prior findings based on the preliminary-stage version of the ITQ (8, 9) and showed that the latent structure of the 12item version of the ITQ effectively captures the distinction between PTSD and DSO symptomatology. The multigroup IRT results showed that the ITQ performed equally well within the community and clinical samples indicating that the scale is appropriate for use in both populations. The newly applied diagnostic algorithm for the optimized ITQ identified an identical number of people qualifying for a diagnosis of PTSD or CPTSD to the preliminary-stage diagnostic algorithm; however, despite the removal of 12 test items from the DSO cluster, the 12-item ITQ identified a slightly higher number of CPTSD cases. Additionally, and in line with previous results (19), individuals who met the criteria for CPTSD based on this new diagnostic algorithm had the highest levels of lifetime interpersonal trauma. This difference was evident in the community and clinical samples; however, the effect was stronger within the clinical sample.

This study had some limitations. First, although the community sample was drawn from a nationally representative panel, it cannot be concluded that the trauma-exposed participant sample itself was nationally representative. Second, the participants in the clinical sample were recruited from centres that provide psychological treatment for trauma exposure and so will not be representative of the help-seeking population in general. Third, these analyses were based on samples drawn from the UK, and, therefore, the generalizability of the current findings to (especially) non-English speaking countries is unknown.

In conclusion, the ITQ is the first instrument designed to capture the ICD-11 PTSD and CPTSD diagnoses. To date, several studies indicated that the preliminary-stage version of the ITQ was a reliable and valid measure of PTSD $(10,11)$ and DSO symptoms (31). This study represents the final development phase in which 12 items have been selected using IRT models based on a trauma-exposed community and clinical sample from the UK population. The findings of the current study indicate that the optimized ITQ, which is now freely available in the public domain, is a valid measure of the ICD-11 PTSD and CPTSD symptoms. Further research is now necessary in order to estimate prevalence rates of PTSD and CPTSD internationally and to identify risk factors for each disorder. The availability of the ITQ will ideally stimulate this important work.

\section{Declaration of interest}

The authors report no conflict of interest relevant to this study.

\section{Disclaimer}

Chris Brewin, Marylène Cloitre and Andreas Maercker participated as members of the World Health Organization Working Group on the Classification of Disorders Specifically Associated with Stress, reporting to the International Advisory Group for the Revision of ICD-10 Mental and Behavioural Disorders. However, the views expressed reflect the opinions of the authors and not necessarily the Working Group or Advisory Group, and the content of this article does not represent WHO policy.

\section{References}

1. World Health Organization. The ICD-10 classification of mental and behavioural disorders: clinical descriptions and diagnostic guidelines. Geneva, Switzerland: World Health Organization; 1992.

2. Keeley JW, Reed GM, Roberts MC, et al. Developing a science of clinical utility in diagnostic classification systems: field study strategies for ICD-11 Mental and Behavioural Disorders. Am Psychol 2016;71:3-16.

3. Maercker A, Brewin CR, Bryant RA, et al. Diagnosis and classification of disorders specifically associated with stress: proposals for ICD-11. World Psychiatry 2013;12:198-206.

4. Brewin CR, Cloitre M, Hyland P, et al. A review of current evidence regarding the ICD-11 proposals for diagnosing PTSD and Complex PTSD. Clin Psychol Rev 2017;58:1-15.

5. American Psychiatric Association. Diagnostic and Statistical Manual of Mental Disorders, 5th edn (DSM-5), 2013.

6. First MB, Reed GM, Hyman SE, Saxena S. The development of the ICD-11 clinical descriptions and diagnostic guidelines for mental and behavioural disorders. World Psychiatry 2015;14:82-90.

7. Cloitre M, Roberts NP, Bisson JI, Brewin CR. The International Trauma Questionnaire (ITQ), 2015. Unpublished Measure.

8. Brewin CR, Lanius RA, Novac A, Schnyder U, Galea S. Reformulating PTSD for DSM-V: life after criterion A. J Trauma Stress 2009;22:366-373.

9. van der Kolk BA, Roth S, Pelcovitz D, Sunday S, Spinazzola J. Disorders of extreme stress: the empirical foundation of a complex adaptation to trauma. J Trauma Stress 2005;18:389-399.

10. Cloitre M, Courtois CA, Charuvastra A, Carapezza R, Stolbach BC, Green BL. Treatment of complex PTSD: results of the ISTSS expert clinician survey on best practices. J Trauma Stress 2011;24:615-627.

11. Karatzias T, Shevlin M, Fyvie C, et al. An initial psychometric assessment of an ICD-11 based measure of PTSD and complex PTSD (ICD-TQ): evidence of construct validity. J Anxiety Disord 2016;44:73-79.

12. Hyland P, Shevlin M, Brewin CR, et al. Validation of posttraumatic stress disorder (PTSD) and complex PTSD using the International Trauma Questionnaire. Acta Psychiatr Scand 2017;136:313-322.

13. Ben-Ezra M, Karatzias T, Hyland P, et al. Posttraumatic Stress Disorder (PTSD) and Complex PTSD (CPTSD) as 


\section{The International Trauma Questionnaire}

per ICD-11 proposals: a population study in Israel. Depress Anxiety 2018;35:264-274.

14. Weathers FW, Blake DD, Schnurr PP, Kaloupek DG, Marx BP, KeANe TM. The life events checklist for DSM-5 (LEC-5), 2013. Instrument available from the National Center for PTSD at www.ptsd.va.gov

15. Ehring T, QuACK D. Emotion regulation difficulties in trauma survivors: the role of trauma type and PTSD symptom severity. Behav Ther 2010;41:587-598.

16. Carlson EB, Waelde LC. The dissociative symptoms scale. Menlo Park, CA: National Center for Posttraumatic Stress Disorder, 1999.

17. Blevins CA, Weathers FW, Davis MT, Witte TK, Domino $\mathrm{JL}$. The posttraumatic stress disorder checklist for DSM5 (PCL-5): development and initial psychometric evaluation. J Trauma Stress 2015;28:489-498.

18. Elklit A, Shevlin M. The structure of PTSD symptoms: a test of alternative models using confirmatory factor analysis. Br J Clin Psychol 2007;46:299-313.

19. Karatzias T, Shevlin M, Fyvie C, et al. Evidence of distinct profiles of Posttraumatic Stress Disorder (PTSD) and Complex Posttraumatic Stress Disorder (CPTSD) based on the new ICD-11 Trauma Questionnaire (ICD-TQ). J Affect Disord 2017;207:181-187.

20. Muthén LK, Muthén BO. Mplus user's guide (7th edn). Muthén \& Muthén, 2013.

21. RAFtery AE. Bayesian model selection in social research. Sociol Methodol 1995;5:111-163.

22. Hu L, BentLer M. Cutoff criteria for fit indexes in covariance structure analysis: conventional criteria versus new alternatives. Struct Equ Modeling 1999;6:1-55.

23. Asparouhov T, Muthén B. Robust chi square difference testing with mean and variance adjusted test statistics. Mplus Web Notes: No. 10. 2006. Available at http://stat model.com/download/webnotes/webnote10.pdf

24. Huggins-Manley AC, HyunSuk H. Assessing the sensitivity of weighted least square model fit indexes to local dependence in item response theory models. Struct Equ Modeling 2017;24:331-340.

25. Chou CP, Bentler PM. Model modification in covariance structure modeling: a comparison among likelihood ratio, lagrange multiplier, and Wald tests. Multivariate Behav Res 1990;25:115-136.

26. YEN WM. Effects of local item dependence on the fit and equating performance of the three-parameter logistic model. Appl Psychol Meas 1984;8:125-145.

27. Chen WH, Thissen D. Local dependence indexes for item pairs using item response theory. J Educ Behav Stat 1997;22:265-289.

28. Saris WE, Satorra A, van der Veld WM. Testing structural equation models or detection of misspecifications? Struct Equ Model 2009;16:561-582.

29. CoHen J. Statistical power analysis for the behavioral sciences. New York, NY: Routledge Academic, 1988.

30. Tanaka J. How big is enough? Sample size and goodnessof-fit in structural equation models with latent variables. Child Dev 1987;58:134-146.

31. Scott KM, Koenen KC, Aguilar-Gaxiola S, et al. Associations between lifetime traumatic events and subsequent chronic physical conditions: a cross-national, cross-sectional study. PLoS ONE 2013;8:e80573.

32. Shevlin M, Hyland P, Roberts NP, Bisson Ji, Brewin CR, Clortre M. A psychometric assessment of Disturbances in Self-Organization symptom indicators for ICD-11 Complex PTSD using the International Trauma Questionnaire. Eur J Psychotraumatol 2018;17:1419749.

\section{Appendix 1. Original ITQ Items}

\begin{tabular}{|c|c|}
\hline Label & Items \\
\hline \multicolumn{2}{|l|}{ PTSD } \\
\hline RE1 & $\begin{array}{l}\text { Having upsetting dreams that replay part of the experience or are } \\
\text { clearly related to the experience }\end{array}$ \\
\hline RE2 & $\begin{array}{l}\text { Having powerful images or memories that sometimes come into your } \\
\text { mind in which you feel the experience is happening again in the } \\
\text { here and now }\end{array}$ \\
\hline RE3 & Being reminded of the experience and then spacing out for a while \\
\hline RE4 & $\begin{array}{l}\text { Having moments when you lost control and acted like you were back } \\
\text { in the experience }\end{array}$ \\
\hline RE5 & $\begin{array}{l}\text { Having a memory of the experience come back to you that was so } \\
\text { strong that you lost track of what was going on around you }\end{array}$ \\
\hline RE6 & $\begin{array}{l}\text { Reacting to people or situations as if you were back in the past } \\
\text { experience }\end{array}$ \\
\hline RE7 & Feeling very upset when something reminded you of the experience \\
\hline RE8 & $\begin{array}{l}\text { Having powerful images or memories that sometimes come into your } \\
\text { mind in which you feel the experience is happening again in the } \\
\text { here and now, even if only for a moment }\end{array}$ \\
\hline AV1 & $\begin{array}{l}\text { Avoiding internal reminders of the experience (for example, thoughts, } \\
\text { feelings or physical sensations) }\end{array}$ \\
\hline AV2 & $\begin{array}{l}\text { Avoiding external reminders of the experience (for example, people, } \\
\text { places, conversations, objects, activities or situations) }\end{array}$ \\
\hline $\mathrm{TH} 1$ & Being 'super-alert', watchful, or on guard \\
\hline TH2 & Feeling jumpy or easily startled \\
\hline \multicolumn{2}{|r|}{ 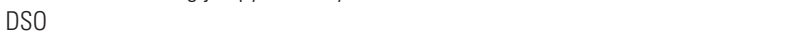 } \\
\hline AD1 & $\begin{array}{l}\text { I react intensely to things that do not seem to affect other people } \\
\text { so much }\end{array}$ \\
\hline AD2 & When I am upset, it takes me a long time to calm down \\
\hline AD3 & My feelings tend to be easily hurt \\
\hline AD4 & I experience episodes of uncontrollable anger \\
\hline AD5 & I do things that people have told me are dangerous or reckless \\
\hline AD6 & I feel numb or emotionally shut down \\
\hline AD7 & $\begin{array}{l}\text { I am the kind of person who has difficulty experiencing feelings of } \\
\text { pleasure or joy }\end{array}$ \\
\hline AD8 & $\begin{array}{l}\text { When I am under stress or confronted with reminders of my trauma, } \\
\text { I often feel that the world is distant or that the world seems different }\end{array}$ \\
\hline AD9 & $\begin{array}{l}\text { When I am under stress or confronted with reminders of my trauma, } \\
\text { I often feel outside my body or feel that there is something strange } \\
\text { about my body }\end{array}$ \\
\hline NSC1 & I feel like a failure \\
\hline NSC2 & I feel worthless \\
\hline NSC3 & I often feel ashamed of myself whether it makes sense or not \\
\hline NSC4 & I feel guilty about things I have done or failed to do \\
\hline DR1 & I feel distant or cut-off from people \\
\hline DR2 & I find it hard to stay emotionally close to people \\
\hline DR3 & I avoid relationships because they end up being too difficult or painful \\
\hline
\end{tabular}




\section{Cloitre et al.}

Appendix 2. International Trauma Questionnaire (ITO)

\section{Instructions}

Please identify the experience that troubles you most and answer the questions in relation to this experience.

Brief description of experience

When did the experience occur? (circle one)

a. less than 6 months ago

b. 6-12 months ago

c. $1-5$ years ago

d. $5-10$ years ago

e. $10-20$ years ago

fmore than 20 years ago

Below are a number of problems that people sometimes report in response to traumatic or stressful life events. Please read each item carefully and then circle one of the numbers to the right to indicate how much you have been bothered by that problem in the past month.

\begin{tabular}{|c|c|c|c|c|c|}
\hline & Not at all & A little Bit & Moderately & Quite a bit & Extremely \\
\hline 1. Having upsetting dreams that replay part of the experience or are clearly related to the experience? & 0 & 1 & 2 & 3 & 4 \\
\hline $\begin{array}{l}\text { 2. Having powerful images or memories that sometimes come into your mind in which you feel } \\
\text { the experience is happening again in the here and now? }\end{array}$ & 0 & 1 & 2 & 3 & 4 \\
\hline 3. Avoiding internal reminders of the experience (for example, thoughts, feelings or physical sensations)? & 0 & 1 & 2 & 3 & 4 \\
\hline $\begin{array}{l}\text { 4. Avoiding external reminders of the experience (for example, people, places, } \\
\text { conversations, objects, activities or situations)? }\end{array}$ & 0 & 1 & 2 & 3 & 4 \\
\hline 5. Being 'super-alert', watchful or on guard? & 0 & 1 & 2 & 3 & 4 \\
\hline $\begin{array}{l}\text { 6. Feeling jumpy or easily startled? } \\
\text { In the past month have the above symptoms: }\end{array}$ & 0 & 1 & 2 & 3 & 4 \\
\hline 7. Affected your relationships or social life? & 0 & 1 & 2 & 3 & 4 \\
\hline 8. Affected your work or ability to work? & 0 & 1 & 2 & 3 & 4 \\
\hline $\begin{array}{l}\text { 9. Affected any other important part of your life such as parenting, or school or } \\
\text { college work, or other important activities? }\end{array}$ & 0 & 1 & 2 & 3 & 4 \\
\hline
\end{tabular}

Below are problems or symptoms that people who have had stressful or traumatic events sometimes experience. The questions refer to ways you typically feel, ways you typically think about yourself and ways you typically relate to others. Answer the following thinking about how true each statement is of you.

\begin{tabular}{|c|c|c|c|c|c|}
\hline How true is this of you? & Not at all & A little Bit & Moderately & Quite a bit & Extremely \\
\hline 1. When I am upset, it takes me a long time to calm down & 0 & 1 & 2 & 3 & 4 \\
\hline 2. I feel numb or emotionally shut down & 0 & 1 & 2 & 3 & 4 \\
\hline 3. I feel like a failure & 0 & 1 & 2 & 3 & 4 \\
\hline 4. I feel worthless & 0 & 1 & 2 & 3 & 4 \\
\hline 5. I feel distant or cut-off from people & 0 & 1 & 2 & 3 & 4 \\
\hline 6. I find it hard to stay emotionally close to people & 0 & 1 & 2 & 3 & 4 \\
\hline 7. Created concern or distress about your relationships or social life? & 0 & 1 & 2 & 3 & 4 \\
\hline 8. Affected your work or ability to work? & 0 & 1 & 2 & 3 & 4 \\
\hline $\begin{array}{l}\text { 9. Affected any other important parts of your life such as parenting, or school or } \\
\text { college work, or other important activities? }\end{array}$ & 0 & 1 & 2 & 3 & 4 \\
\hline
\end{tabular}

